\title{
REGISTER
}

\section{Sach- und Ortsregister}

Verzichtet wurde auf die Nennung kleinerer Dörfer, Gehöfte und Forts um Metz sowie auf Orte, deren Erwähnung nichts zur Sache tut. Eine topographische Orientierungshilfe zur Gegend um die Festung bieten die Karten: Abb. 6 (S. 32), 9 (S. 58) und 12 (S. 84). Mars-la-Tour und Gravelotte-St. Privat wurden in der Regel nur im unmittelbaren Zusammenhang mit den Schlachten vom 16. bzw. 18. August 1870 registriert. Eine hochgestellte Ziffer im Register verweist auf die Fußnote auf der angegebenen Seite. Steht ein + davor, bezieht sich der Registerbegriff auf die Seite und die Fußnote.

Adel, preußischer $37,39,41,43 \mathrm{f} ., 113-$ $121,122^{+47 / 50 / 51}, 123$

Antisemitismus/Dolchstoßlegende 30, $118 \mathrm{f}$.

Artillerie/Feuerwalze/Trommelfeuer $39-41,57,59,86,103,134$

Austrasien 12

Azincourt (Schlacht 25.10.1415) $12^{8}$

Bad Ems 23

Bahntransporte/Sanitätszüge $21,52,68-$ 70

Bazeilles (bei Sedan) 90, 93

Befreiungskriege 25, 43, 92, 116

Belagerung/Belagerungsalltag 6,59-82, $126 \mathrm{f}$.

Belfort 17, 129+17

Bouvines (Schlacht 27.7. 1214) $6^{24}, 12^{8}$, 37,55

Chassepot $34,39,59,97$

Chislehurst 76

Chloroformnarkose 51

Colombey-Nouilly (Schlacht 14. 8. 1870, frz. auch Borny) 31, 37, 4352, 44

Courcelles 69, 83, 108

Danzig 9

Deutscher Sonderweg 122

Diedenhofen 85

Dolchstoßlegende (s. Antisemitismus)

Dorn 110

Dünkirchen 17

Elsaß-Lothringen (Reichsland) 2-4, $24 \mathrm{f} ., 64,107,119,127-135$

Epidemien $52,66^{+43}, 71,75^{+92}, 126$
Épinal 129

Erster Weltkrieg (s. Stellungskrieg)

Feldgeistliche $50 \mathrm{f}$.

Feldhyänen 50, 53

Feuerwalze (s. Artillerie)

Flucht/Flüchtlinge $11^{+4}, 15,30,73,132$

Franctireurwesen/Geißelerschießungen $73,87-89,90^{+39}, 91 \mathrm{f} ., 126$

Frauen/weiblicher Patriotismus 6, 9296, 111, 134

Friedens-/Kapitulationsverhandlungen $76^{+99}, 99 f ., 103-105$

Friedensvertrag:

- Frankfurter $127 \mathrm{f}$.

- Versailler 128, 130

Gefangenschaft/Gefangenentransport $68,108,110,126 \mathrm{f}$.

Geißelerschießungen (s. Franctireurwesen)

Gentry 122

Gerechter Krieg 51

Geschlechtskrankheiten (s. Prostitution)

Goldene Bulle 24

Gorze 49+71, 50, 71 f., 9982

Grande Peur 30+46

Gravelotte-St. Privat (Schlacht 18. 8. 1870) $5,31,38-40,41^{+46}, 42,43^{+52 / 54}$, $44 \mathrm{f} ., 49,54 \mathrm{f} ., 74,115,125^{+1}, 126,131$, 134

Hannover $111^{+38}$

Heimatfront 7,23

Heeresgruppe Châlons 31, 57, 83

Hohenzollern (s. Preußen)

Hunger/Versorgungsnöte $13,15,66^{+43}$, 
67-70, 75f., 78-80, 84, 97-99, 103, 105, $108,111,126 \mathrm{f}$.

Hunnen 12, 125

Ideen von 1914/Kulturkrieg 89,135

Jena $17,26,55^{100}, 121,134$

Jerusalem 9

Kaiserproklamation/Reichsgründung 56, 121, 127

Kassel 76, 110

Katholizismus $12,15^{+20}, 117,132^{+34}, 133$

Kavallerie/Ulanen 27, 34-37, 69f., 78, 88,103

Kirche (s. Religion)

Koblenz 19, 110

Kolberg 9

Konstantinopel 9

Kriegsziele $25 \mathrm{f} ., 107,119$

Krimkrieg/Sewastopol 9f., 21, 25, 59, $74,76^{96}, 84$

Kulturkrieg (s. Ideen v. 1914)

Landau 26

Landserjargon/„Lyrik von unten“ 53 , 67,89

Langemarck-Mythos 44

Lazarette/Wundbehandlung $45^{+60}, 51 \mathrm{f}$, $71 \mathrm{f} ., 74,75^{+92}$

Legano 10

Leningrad 9

Lineartaktik $39 \mathrm{f}$.

Loire/Loire-Feldzug 46, 88, 92

Lothringen (s.a. Elsaß-Lothringen.) 11$18,24,53-55,89,92^{46}, 97$ f., $107,127-$ $130,132,135$

Mars-la-Tour (Schlacht 16.8. 1870) 5, $33-39,44,55,74,125^{+1}, 126,132$

Mantua $9 \mathrm{f}$.

Mercy le Haut/Peltre/Courcelles (Ausfälle, 23. bzw. 27. 9. 1870) $57,83 \mathrm{f}$.

Metz:

- Garnison/Festung 12-19, 73 f., 125136

- Reichsstadt 12-14,24,

- Zustände-/Unruhen in der Stadt 74$82,105-107^{+20}, 111 \mathrm{f} ., 131^{+27}, 132$

- deutsche Gefangene $80 \mathrm{f} ., 126$

Militarismus $35-37,117,121 \mathrm{f} ., 131,135$ Montmédy 83
Moskau $63^{29}$

Musik/Militärkapellen/Marseillaise 21, $44,68+58,78,85,103,106^{20}, 117,133$

Nahkampf $40 \mathrm{f}, 59,86+15$

Nancy $11,12^{8}, 29,71,129,131 \mathrm{f}$.

Nation/Nationalismus 2, 6-10,13, 21, 23-25, 35, 40, 4348, 64, 68, 89, 93 f., $101^{+90}, 113 \mathrm{f} ., 116^{+21}, 117-119,129-$ 136

Natürliche Grenzen (limites naturelles) $24^{+11}, 25$

Noisseville-St. Barbe (Schlacht 31. 8/1. 9. 1870) $82-87$

Paris 9, 11, 23, 26f., 30, 37, 57+2, $63+30,64,76$ f., 81 f., 87,94 f., $101^{89}, 107$, $116,121,127$

Patriotismus, weiblicher (s. Frauen)

Patrouillen (s. Vorpostendienst)

Perceurs 106

Peschiera 10

Pferde $36^{+23 / 25}, 69 \mathrm{f}$., $78 \mathrm{f}$., 111

Pont-à-Mousson 4971, $70 \mathrm{f}$.

Port Arthur 9

Potsdam 64, $115 \mathrm{f}$.

Preußen/Hohenzollern (Dynastie) 23, $26+31,33$ f., 39f., 60, 77, 95, 107, 119, 121-123

Propaganda 88f., 93, 101, 128

Prostitution/Sexualität/Geschlechtskrankheiten $94^{+61}, 95 \mathrm{f}$.

Protestantismus 115, 117, 132

Provinces perdues 55

Regen u. Schlamm (s. Wetter)

Reichsgründung (s. Kaiserproklamation)

Reichsland (s. Elsaß-Lothringen)

Reims 129

Religion/Kirche 6, 94, 117, 118+26, 127

Remilly $69 \mathrm{f}$.

Roßbach (Schlacht 5. 11. 1757) 37

Rheinarmee $11,29,33,38,44,57,76^{+98}$, $79,83-85,87,98^{78}, 99,103,105,107 \mathrm{f}$., $110,125-128$

Sadowa (Schlacht 3. 7. 1866, dt. Königgrätz) $16^{+29}, 29$

Saarbrücken $11,27,29,68,70,84,108^{28}$, 131

Saarlouis 71, 85, 108

Sanitätszüge (s. Bahntransporte)

Schlettstadt 80

Schlieffen-Plan 130 
Sedan (Schlacht 1./2. 9. 1870) 2,57,68, $76,81,84,85^{11}, 87,93,97,108,128,135$

Sewastopol (s. Krimkrieg)

Sexualität (s. Prostitution)

Soldat citoyen 116

Soldatische Männlichkeit 38f., 43, 51, 61

Spichern/Forbach (Schlacht 6. 8. 1870) $29,66^{44}$

Spleen (Langeweile) 64, 127, 132

Stalingrad (Schlacht Sept. 1942 bis Febr. 1943) 108

St. Remy/Woippy (Ausfälle 2. bzw. 7.10. 1870) $57,83,105^{11}$

Stellungskrieg/Erster Weltkrieg 41, 45, 58-60, 87, 96-99, 108, 127, 130

Sterben (s. Verwundetsein)

Straßburg $9,25,29,57,64^{+32}, 75,80$, $128,134 f$.

Tannenberg (Schlacht 26.-31. 8. 1914) 108

Thionville 106

Totaler Krieg 125

Totenbestattung/Totenkult $2 \mathrm{f},{ }^{+7}, 51-56$, $81,131,135$

Toul $13,80,129$

Tours 82, $110^{36}$

Trier 12,70

Trommelfeuer (s. Artillerie)
Ulanen (s. Kavallerie)

Uniform 60f., 96f., 110, $115 \mathrm{f}$.

Valmy (Kanonade 20.9.1792) 55

Verdun 33, 37, $129 \mathrm{f},{ }, 134$

Vernéville 62, 113

Verona 10

Versailles 2, 56, 76 $6^{99}, 101^{89}, 125,128$

Versorgungsnöte (s. Hunger)

Verwundetsein/Sterben 45-51

Volkskrieg $5^{22}, 89 \mathrm{f} ., 91^{+44}, 92 \mathrm{f}$.

Vorpostendienst/Patrouillen 59, 96-99

Weißenburg (Schlacht 4.8. 1870) 24, 29

Wetter/Regen u. Schlamm 1,63f., 69, $72 \mathrm{f} ., 100^{85}, 105,107 \mathrm{f}$.

Waterloo (Schlacht 18.6. 1815) 128, 33

Wehrpflicht, allgemeine 26

Willensmenschen 116,121

Wörth/Reichshofen (Schlacht 6. 8. 1870) 29,36

Wundbehandlung (s. Lazarette)

Zivilbevölkerung $6 f ., 15,79+113,97 \mathrm{f}$., $100 \mathrm{f}, 125 \mathrm{f} ., 135 \mathrm{f}$.

Zündnadelgewehr 39 